\title{
Malakoplakia and adenocarcinoma of the caecum: a rare association
}

D Sandmeier, L Guillou

\begin{abstract}
Colonic malakoplakia can occur either as a part of systemic disease with multiple colonic and extracolonic deposits or as an incidental finding associated with a rectal or sigmoidal adenocarcinoma. A case is reported which shows an association between malakoplakia and adenocarcinoma of the caecum.
\end{abstract}

(F Clin Pathol 1993;46:959-960)

\section{Case report}

A 74 year old non-immunocompromised man was admitted because of diffuse abdominal pain of several months' duration, which had recently been accompanied by episodes of diarrhoea. A medical history consisted of Parkinson's disease treated with prednisolone $(5 \mathrm{mg} /$ day $)$ and levodopa $(3 \times 100 \mathrm{mg} /$ day $)$. There was neither rectal bleeding nor weight loss and abdominal palpation was unremarkable. A gastrographic enema showed a bulky neoplasm in the caecum and sigmoidal diverticulosis. An abdominal sonogram did not show liver metastases. A right hemicolectomy was performed. The postoperative course was uneventful.

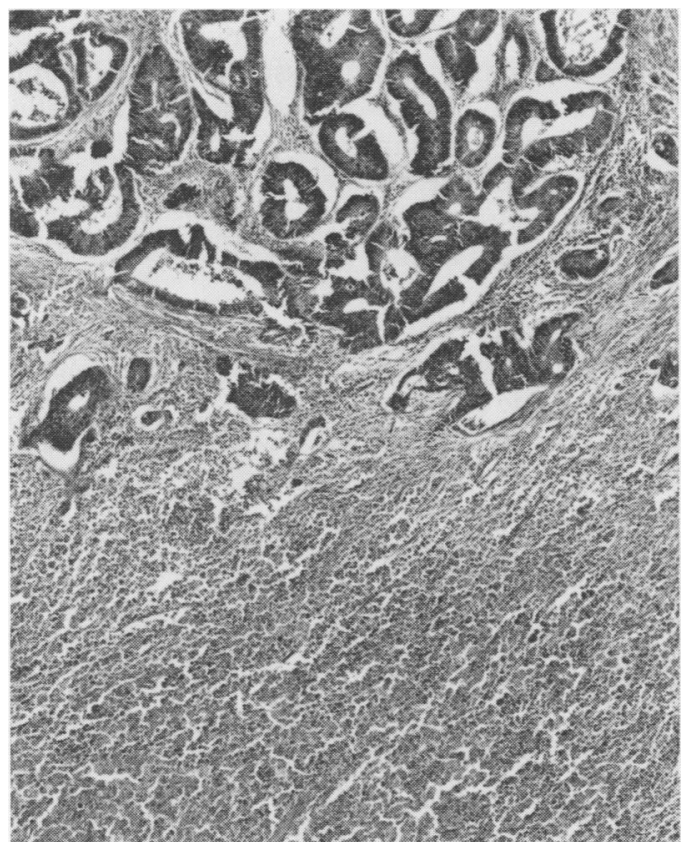

University Institute of Pathology, Lausanne, Switzerland D Sandmeier L Guillou

Correspondence to:

Dr Louis Guillou, Institu Universitaire de Pathologie, 25 rue de Bugnon,

1011 Lausanne, Switzerland

Figure 1 The soft nodule consisted mainly of sheets of benign looking large histiocytes and it was found close to the caecal adenocarcinoma (haematoxylin and eosin).

\section{Pathological findings}

Grossly, the polypoid and circular caecal tumour measured $6.5 \times 10.5 \mathrm{~cm}$. It had penetrated the bowel wall reaching the mesocolic fat. The mesocolon contained a soft, well delineated, whitish nodule measuring $1 \mathrm{~cm}$ in diameter, believed to represent a metastatic lymph node. There was no associated right colonic diverticulosis.

Histological examination showed an ulcerated well differentiated adenocarcinoma of the caecum. Three pericolic lymph nodes were positive for metastatic disease. The tumour was staged Dukes C, pT3N1Mx (TNM classification, 1987). The soft nodule corresponded to a collection of histiocytes that exhibited abundant eosinophilic, somewhat granular cytoplasm (fig 1). Several of these histiocytes, especially those close to the neoplastic infiltration, contained intracytoplasmic round bodies of varying size which reacted positively with the periodic acid Schiff, with and without diastase predigestion, iron (Prussian blue), and calcium (Von Kossa) stains (fig 2). Gram, Ziehl, and methenamine silver (Grocott) stains were negative. Examination under polarised light failed to show any birefringent foreign material. At the edges of the nodule, histiocytes

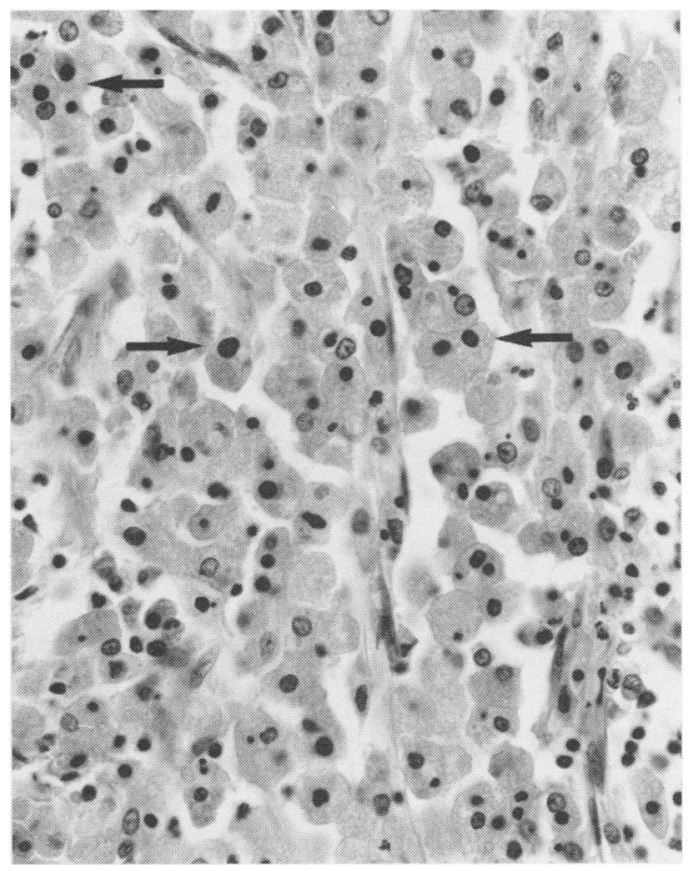

Figure 2 The histiocytes had an abundant slightly granular cytoplasm that frequently contained round calcified Michaelis-Gutmann bodies (arrows)
Neutrophilic polymorphonuclear cells, lymphocytes, and plasmocytes were also mixed with the histiocytic population (Von Kossa stain). 
were mixed with numerous lymphocytes, plasmocytes, and neutrophilic polymorphonuclear cells. The intracytoplasmic, siderocalcific, round bodies were interpreted as Michaelis-Gutmann bodies and caecal adenocarcinoma with coexistent malakoplakia was diagnosed. Regional lymph nodes did not show evidence of malakoplakic deposits.

Ultrastructural examination performed on formalin fixed material showed that the histiocytic cells had indented nuclei with marginated chromatin. The cytoplasm was abundant and contained numerous primary lysosomes and phagolysosomes. The latter were of varying size and most of them contained laminated structures resembling myelin figures. Some of the largest lysosomes exhibited progressive central calcification ending in the formation of typical membranebound Michaelis-Gutmann bodies measuring up to $3.5 \mu \mathrm{m}$ in diameter. Intra- or extracellular bacteria were not observed.

\section{Discussion}

Malakoplakia, first described by Michaelis and Gutmann in 1902, occurs predominantly in the genitourinary tract and less frequently in the gastrointestinal tract $(11 \%$ of the cases according to $\mathrm{McClure}^{1}$ ). Malakoplakia has been reported to occur in conjunction with tumoral (lymphoma, colonic adenomas, and adenocarcinomas) and non-tumoral condition (tuberculosis, sarcoidosis, malnutrition, diabetes mellitus, alcoholic liver disease, treatment with steroids and other immunocompromising states). Fifteen cases of malakoplakia associated with colonic adenocarcinoma have been recorded to date. ${ }^{1-4}$ All of the tumours were located in the rectosigmoid region. To our knowledge, the present case would be the first to record such an association in the caecum.

In 1983, Eile et $a l^{2}$ reviewed the published findings and suggested that cases of colonic malakoplakia could be divided into two groups on the basis of distinctive clinicopathological features. In one group, made up initially of 12 cases to which a further five can be added ${ }^{134}$ the malakoplakic process represented a localised phenomenon closely associated with a tumour. The neoplasm consisted of a colonic or rectal adenocarcinoma in 15 cases and of a colonic adenoma in two cases. All of the tumours were found in the rectosigmoid region and were responsible for the presenting symptoms. Malakoplakic deposits were incidental findings and they were entirely removed at surgery. Spread outside the gastrointestinal tract occurred very rarely. The patients were often in their seventh or eighth decades and the male to female ratio was $2: 1$. Underlying acquired pathological conditions (autoimmune disease and immunocompromising states) were rarely found and malakoplakia seemed to have no influence on the course of the colonic carcinoma. ${ }^{4}$ Our case clearly belongs to that group. On the other hand, in the second group of 28 cases, malakoplakia was not associated with any colonic tumour. Although the rectosigmoid was the most common site, in half of the cases there were also extra-colonic deposits (regional lymph nodes, urinary tract, peritoneum, etc) and underlying systemic disease (tuberculosis, alcoholic liver disease, immune deficiency, etc) in $65 \%$ of the patients.

The pathogenesis of malakoplakia and the origin of Michaelis-Gutmann bodies is not completely understood, although the disease may be considered to be the result of an impairment of the mononuclear phagocyte and immunoregulatory effector system. ${ }^{1}$ In fact, partly disintegrated bacteria have been occasionally observed in the phagolysosomes of the macrophages (von Hansemann's cells) at the ultrastructural level; most of them have been coliform bacilli, but mycobacteria and fungi have also been found. ${ }^{5}$ Similarly, several recent reports ${ }^{5}$ have focused on an unusual association between Rhodococcus equi infection and pulmonary malakoplakia in patients with AIDS. Extensive malakoplakia lesions suggest generalised immune dysfunction, but the colonic carcinoma associated malakoplakia seems to represent a localised phenomenon resulting only from a deficient local immune host response, possibly related to the presence of the neoplasm. This local change in immunity may involve two mechanisms: facilitation of the proliferation of coliform bacteria and modification of the phagocytic abilities of the macrophage. In the case reported here the prednisolone given to the patient might have played a significant role in the pathogenesis of the malakoplakia. Neither in our case nor in the reported cases of carcinomaassociated malakoplakia, however, was the presence of intracellular bacilli documented.

We thank Sophie Simon Valla, MD for performing the ultrastructural study and J Maillardet and S Burki for photographic assistance.

1 McClure J. Malakoplakia. f Pathol 1983;140:275-330.

2 Elie $\mathrm{H}$, Maupin JM, Mandard JC. Malacoplasie associée à un adénocarcinome rectal. Revue de la littérature. À propos d'un cas. Ann Pathol 1983;3:333-6.

3 Moran CA, West B, Schwartz IS. Malakoplakia of the colon in association with colonic adenocarcinoma. $A m \mathcal{F}$ Gastroenterol 1989;84:1580-2.

4 Letessier E, Cuillière P, Lehur PA, et al. Malacoplasie associée à un adénocarcinome colique. Revue de la littérature. A propos d'un cas. Ann Chir 1987;4:534-6.

5 Schwartz DA, Ogden PO, Blumberg HM, Honig E. Pulmonary malakoplakia in a patient with the acquired immunodeficiency syndrome. Differential diagnostic considerations. Arch Pathol Lab Med 1990;114:1267-72. 